\title{
Produção de aventais descartáveis em complexo prisional: ação de enfermagem no enfrentamento à COVID-19
}

Production of disposable gowns in a prison complex: nursing action in confronting COVID-19

Producción de batas desechables en complejo carcelario: acción de enfermería en el enfrentamiento a la COVID-19

\section{George Oliveira Silva ${ }^{\mathrm{I}}$, Larissa Viana Ues ${ }^{\mathrm{II}}$, Carlos Gustavo Martins Hoelzel ${ }^{\mathrm{III}}$, Heliny Carneiro Cunha Neves ${ }^{\mathrm{IV}}$, Katiane Martins Mendonça ${ }^{\mathrm{v}}$, Luana Cássia Miranda Ribeirovi}

\begin{abstract}
Resumo: Objetivo: compartilhar a experiência relacionada à produção de aventais descartáveis conduzida pela enfermagem em complexo prisional no contexto da pandemia da COVID-19. Método: relato de experiência das ações de extensão de projeto de produção de equipamentos de proteção individual realizadas em complexo prisional da região Centro-Oeste do Brasil, no período de março a junho de 2020. Resultados: foram produzidos 6.000 aventais, adotando as normas da Associação Brasileira de Normas Técnicas. No processo, houve aprendizado das medidas de higiene e limpeza para prevenção da COVID-19, com destaque para a higienização das mãos, readequação de fluxo e do ambiente de produção. Conclusão: a experiência pautou-se no compromisso social da universidade pública e foi oportunidade ímpar de desenvolvimento de conhecimentos e habilidades, bem como de reeducação e ressocialização dessa população. Além disso, reforçou o papel da enfermagem no enfrentamento de agravos de saúde pública.
\end{abstract}

Descritores: Equipamento de Proteção Individual; Infecções por Coronavírus; Pandemias; Relações ComunidadeInstituição; Pessoas Privadas de Liberdade

Abstract: Objective: to share the experience related to the production of disposable gowns conducted by nursing staff in a prison complex in the context of the COVID-19 pandemic. Method: experience report of project extension

\footnotetext{
I Enfermeiro, Mestre em Enfermagem, Universidade Federal de Goiás, Goiânia, Goiás, Brasil. E-mail: georgeoliveira.z9@gmail.com, Orcid: http://orcid.org/0000-0001-9863-3161

II Enfermeira, Universidade Federal de Goiás, Goiânia, Goiás, Brasil. E-mail: larissaues1@gmail.com, Orcid: http://orcid.org/0000-0003-2418-1355

III Desenhista Industrial, Doutorado em Engenharia de Produção, Universidade Federal de Goiás, Goiânia, Goiás, Brasil. E-mail: carlos.gustavo.ufg@gmail.com, Orcid: http://orcid.org/0000-0003-3136-7270

IV Enfermeira, Doutora em Enfermagem, Universidade Federal de Goiás, Goiânia, Goiás, Brasil. E-mail: nynne_cunha@yahoo.com.br, Orcid: http://orcid.org/0000-0001-8240-1059

V Enfermeira, Doutora em Enfermagem, Universidade Federal de Goiás, Goiânia, Goiás, Brasil. E-mail: katiane.martins@ufg.br, Orcid: http://orcid.org/00000003-2266-6383
}

VI Enfermeira, Doutora em Enfermagem, Universidade Federal de Goiás, Goiânia, Goiás, Brasil. E-mail: luaufg@yahoo.com.br, Orcid: http://orcid.org/00000002-4254-2030 
Produção de aventais descartáveis em complexo prisional: ação de enfermagem no enfrent... $\mid 2$

actions for the production of personal protective equipment carried out in a prison complex in the Center-West region of Brazil, from March to June, 2020. Results: a total of 6,000 gowns were produced, adopting the standards of the Brazilian Association of Technical Standards. In the process, there was learning about hygiene and cleaning measures to prevent COVID-19, with emphasis on hand hygiene, readjustment of flow and of the production environment. Conclusion: the experience was based on the social commitment of the public university and was a unique opportunity to develop knowledge and skills, as well as of re-education and re-socialization of this population. In addition, it strengthened the nursing role in dealing with public health problems.

Descriptors: Personal Protective Equipment; Coronavirus Infections; Pandemics; Community-Institutional Relations; Prisoners

Resumen: Objetivo: compartir la experiencia de producción de batas desechables, manejada por la enfermería en complejo carcelario en el contexto de la pandemia de COVID-19. Método: relato de experiencia de las acciones de extensión de proyecto de producción de equipos de protección individual realizadas en complejo carcelario de la región Centro-Oeste de Brasil, en el período de marzo a junio de 2020. Resultados: fueron producidas 6.000 batas, adoptando las normas de la Asociación Brasileña de Normas Técnicas. Durante el proceso, hubo aprendizaje de las medidas de higiene y limpieza para prevención de la COVID-19, con destaque para la higienización de las manos, readecuación del flujo y del ambiente de producción. Conclusión: la experiencia se basó en el compromiso social de la universidad pública y fue una gran oportunidad de desarrollo de conocimientos y habilidades, además, fomentó la reeducación y resocialización de esa población. Por fin, reforzó el rol de la enfermería en el enfrentamiento de situaciones graves de salud pública.

Descriptores: Equipo de Protección Personal; Infecciones por Coronavirus; Pandemias; Relaciones ComunidadInstitución; Personas Privadas de Libertad

\section{Introdução}

O relato de uma série de casos de pneumonia de origem desconhecida em dezembro de 2019 na cidade de Wuhan, China, despertou atenção mundial. Em janeiro do ano seguinte o mapeamento genético do genoma viral do então intitulado SARS-CoV-2 revelou ao mundo um marco epidemiológico com o aumento de infecções por esse vírus e a consequente pandemia de Coronavirus Disease 2019 (COVID-19), deflagrada em março de 2020. ${ }^{1-2}$ Trata-se de uma síndrome respiratória transmitida por via direta - propagação pelo indivíduo contaminado de gotículas advindas de tosse ou espirro - ou indireta, contato com superfícies e objetos contaminados. ${ }^{2}$

Os sistemas de saúde do mundo todo, especialmente dos países em desenvolvimento, têm sido desafiados com o aumento exponencial do número de casos, potencializados por fatores 
sociais, econômicos e políticos, revelando-se uma das mais graves pandemias enfrentadas pela humanidade. $^{3}$ A demanda de vagas em instituições hospitalares tem aumentado significativamente, o que implica em ocupação dos leitos gerais e de Unidades de Terapia Intensiva (UTI), uso de equipamentos tecnológicos e a atuação de profissionais de saúde. ${ }^{4}$

Diante da inexistência de tratamento medicamentoso eficaz, as formas mais efetivas de combate à COVID-19 consistem na vacina e na ruptura de sua cadeia de transmissão. Portanto, as medidas de prevenção embasadas em evidências científicas e orientadas à população no combate à doença têm sido, além da vacina, o distanciamento social, higiene de mãos, etiqueta respiratória e uso de Equipamento de Proteção Individual (EPI), principalmente o uso de máscaras. ${ }^{2}$

No contexto da atenção à saúde, os EPIs constituem barreiras de proteção que evitam a exposição da pele, vias aéreas, membranas mucosas e vestuário a agentes infecciosos. ${ }^{5}$ Portanto, são primordiais na prevenção da transmissão do SARS-CoV-2 e outros microrganismos, pois ao serem utilizados de forma combinada e associados às demais precauções-padrão conferem proteção ao profissional de saúde na assistência. ${ }^{5-6}$

Dada a alta demanda de atendimentos nos serviços de saúde e consequente aumento do uso de EPI, evidenciou-se a dificuldade da indústria em fornecer esses recursos em tempo hábil, com qualidade e eficiência necessárias, ${ }^{5,7}$ instituindo assim um alerta sobre a escassez mundial desses equipamentos de proteção. Adicionalmente, é evidente que tal agravo pode influenciar diretamente os profissionais de saúde, colocando-os em risco de infecção pelo SARS-CoV-2, dentre outros patógenos, além de impactar na segurança do paciente. ${ }^{8}$

Dessa forma, a Organização Mundial da Saúde (OMS) tem orientado o gerenciamento adequado do uso de EPI, por meio de ações como uso racional para cada procedimento e profissão, bem como monitoramento de seu quantitativo e controle de distribuição. ${ }^{5}$ Esse cenário é especialmente vivenciado em países em desenvolvimento, os quais ainda enfrentam desafios 
Produção de aventais descartáveis em complexo prisional: ação de enfermagem no enfrent... $\mid 4$

econômicos e sociais, dificultando a execução de medidas de prevenção e combate à COVID-19, somado à falta de infraestrutura dos serviços de saúde para o atendimento da população. ${ }^{3}$

No que diz respeito aos profissionais de saúde, o déficit global no fornecimento de EPI está diretamente associado à segurança ocupacional, ${ }^{3,9}$ sendo observadas altas taxas de infecção que variam de $3 \%$ a $29 \%{ }^{10}$ Assim, faz-se necessário preservar a segurança desses profissionais diante da alta carga de exposição e transmissibilidade do vírus. ${ }^{6-7}$

Em decorrência da nova demanda, o Ministério da Saúde e a Agência Nacional de Vigilância Sanitária (Anvisa) promulgaram, de forma extraordinária e temporária, a Resolução de Diretoria Colegiada (RDC) nº 356 de 23 de março de 2020 que trata sobre as condições de fabricação e aquisição de produtos hospitalares para o uso das instituições de saúde durante o período da pandemia, permitindo a confecção de EPI, tais quais: máscaras cirúrgicas; óculos de proteção; protetores faciais; vestimentas hospitalares descartáveis; gorros e propés. ${ }^{11}$

A partir desse marco, diversas instituições públicas, privadas e organizações não lucrativas têm produzido EPI, com vistas a suprir as demandas dos serviços de saúde e evitar a escassez. $^{12}$ Assim, este estudo teve como objetivo compartilhar a experiência relacionada à produção de aventais descartáveis conduzida pela enfermagem em complexo prisional no contexto da pandemia da COVID-19.

\section{Método}

Relato de experiência das ações de extensão do Projeto EPI-UFG realizadas em um complexo prisional da região de Goiânia, no período de março a junho de 2020, pela Universidade Federal de Goiás (UFG) em parceria com a Diretoria Geral de Administração Carcerária (DGAP) do Estado de Goiás. A partir de uma robusta estrutura com parcerias internas e externas à comunidade universitária para o combate à COVID-19, a UFG deu início 
ao projeto sob liderança da Reitoria da UFG e das unidades acadêmicas da Faculdade de Enfermagem e Faculdade de Artes Visuais.

O projeto consistiu na estruturação de um centro de produção e distribuição de máscaras e aventais descartáveis de tecido não tecido (TNT) para atender às demandas dos serviços de saúde do Estado de Goiás e de populações vulneráveis no período da pandemia. Além disso, dispôs de etapa de capacitação de voluntários (estudantes, professores, indivíduos da comunidade e reeducandos) em relação às medidas de higiene e segurança para a produção dos EPIs, em atendimento às condutas básicas preconizadas pela Associação Brasileira de Normas Técnicas $(\mathrm{ABNT})$ quanto à produção e especificações. ${ }^{13}$ Esse relato restringiu-se ao objetivo de produção de aventais descartáveis de uso hospitalar do Projeto EPI-UFG, o qual seguiu as especificações técnicas descritas no Quadro 1.

Quadro 1 - Especificações técnicas dos aventais de procedimentos. Goiânia, GO, Brasil, 2020.

\begin{tabular}{|c|c|c|}
\hline \multirow{2}{*}{ Descrição do produto } & \multicolumn{2}{|r|}{ Insumos } \\
\hline & Item & Produto \\
\hline \multirow{7}{*}{$\begin{array}{l}\text { Avental de procedimentos hospitalares em } \\
\mathrm{TNT}^{*} 50 \mathrm{~g} / \mathrm{m}^{2} \text { e } 60 \mathrm{~g} / \mathrm{m}^{2} \quad(100 \% \\
\text { polipropileno), de corte reto, painel } \\
\text { inteiriço } 1 / 2 \text { costas/frente } / 1 / 2 \text { costas, com } \\
\text { mangas longas, cinto e gola padre } \\
\text { amarrada atrás. O avental é liso (fechado) } \\
\text { na frente e transpassado } 5,0 \mathrm{~cm} \text { nas costas } \\
\text { com amarração na altura da cintura e na } \\
\text { gola. O punho é ajustado com elástico. }\end{array}$} & Tecido & TNT $40 \mathrm{~g} / \mathrm{m}^{2}$, cor branca, $2 \mathrm{~m}$ \\
\hline & Linhas & $\begin{array}{l}\text { Fio de overlock POLLY PT05C } 70 \mathrm{~g} \text { para ponto } \\
\text { corrente Classe } 504 \text {, cor branca, } 7,9 \mathrm{~m}\end{array}$ \\
\hline & & $\begin{array}{l}\text { Linha para ponto corrente Classe } 504 \text { e ponto Classe } \\
301 \text {, cor branca, } 16,45 \mathrm{~m}\end{array}$ \\
\hline & Aviamentos & $\begin{array}{l}15 \mathrm{~cm} \text { de elástico de } 5 \mathrm{~mm} \text { para tamanho } \mathrm{G} \text {, cor branca, } \\
\text { para os punhos }\end{array}$ \\
\hline & & $\begin{array}{l}13 \mathrm{~cm} \text { de elástico de } 5 \mathrm{~mm} \text { para tamanho } \mathrm{M} \text {, cor branca, } \\
\text { para os punhos }\end{array}$ \\
\hline & & Carimbo etiqueta de composição e de tamanho \\
\hline & & Carimbo de etiqueta da marca UFG \\
\hline
\end{tabular}

${ }^{*}$ Tecido não tecido.

Fonte: Manual do projeto EPI-UFG [Ebook] (UFG, 2020).

Participaram dessa etapa do projeto: docentes do curso de Graduação em Enfermagem da UFG que elaboraram e implementaram o protocolo de biossegurança, além de terem garantido a adequação dos EPI às normas da ABNT (assessoria) e usabilidade (relação do EPI produzido com a atividade a ser executada); docentes dos cursos de Graduação em Design, Moda e 
Produção de aventais descartáveis em complexo prisional: ação de enfermagem no enfrent... |6

Engenharia Têxtil da UFG que participaram das etapas de análise da matéria-prima, modelagem e organização do processo de produção; dois enfermeiros estudantes da pós-graduação stricto sensu que implementaram o protocolo de biossegurança e supervisionaram a produção in loco; além da equipe operacional, composta por 15 reeducandos do sexo masculino e do regime fechado, atuantes na indústria do complexo prisional, fornecidos pela DGAP, que participaram ativamente na confecção dos aventais. Não foram estabelecidos critérios de inclusão para participação nas ações do projeto.

A execução do projeto ocorreu de acordo com as seguintes etapas: 1. Estabelecimento da parceria com a DGAP para a produção de aventais; 2 . Adaptação do protocolo de boas práticas e segurança à estrutura do local de produção no complexo; 3. Capacitação da equipe operacional acerca das medidas de segurança, protocolo e orientações quanto ao modelo e processo de costura; 4. Organização e limpeza do ambiente de trabalho realizada pelos reeducandos e supervisionada pela equipe do projeto; 5. Processo de produção; 6. Processo de dobradura e empacotamento; e 7. Processo de transporte e guarda dos aventais em área exclusiva.

Compreendendo o risco de contaminação pelo SARS-CoV-2 e a vulnerabilidade dos reeducandos no contexto prisional, foram adotadas as medidas de prevenção à COVID-19 preconizadas nacional e internacionalmente. Em todas as etapas houve supervisão de membros da equipe do projeto (professores e enfermeiros), tendo sido seguidos os protocolos definidos para a produção dos aventais. ${ }^{13}$

Os resultados do presente estudo estão vinculados às atividades das ações de extensão do Projeto EPI-UFG (PJ105-2020). Devido a se tratar de um relato de experiência, não houve necessidade de aprovação por Comitê de Ética em Pesquisa; no entanto, as instituições envolvidas e os estudantes foram resguardados em sua individualidade.

\section{Resultados da experiência}


A experiência no complexo prisional resultou na produção de 6.000 aventais, auxiliando no fornecimento de EPI para combate à COVID-19. Para tal, foi realizada inicialmente a análise da matéria-prima para a produção dos aventais e o modelo seguiu as normas da ABNT. ${ }^{14}$

Tais ações corroboram com estratégias internacionais adotadas diante da escassez de EPI vivenciada durante a pandemia. ${ }^{9,15}$ Assim, surgem novas alternativas à fabricação e distribuição de tais insumos para suprir as necessidades dos serviços de saúde com bom custo-benefício que podem ser implementadas especialmente em países em desenvolvimento..$^{9,15-17}$

Nesse contexto, a Universidade tem promovido impactos positivos à sociedade por meio de divulgação científica, ações extensionistas diversas, desenvolvimento de tecnologias, intervenção direta na sociedade, difusão de informações, produção de estudos de alcance internacional e participação na rede de suprimentos, ${ }^{12,18}$ além de ações promovidas pela experiência relatada de importância para a manutenção da estrutura do sistema de saúde.

\section{Capacitação da equipe operacional, organização, gestão do processo e ambiente de trabalho}

A rápida mobilização para a implementação da proposta dada a alta demanda de EPI trouxe desafios inerentes ao ambiente prisional. Dentre os desafios vivenciados destacaram-se a infraestrutura da sala de confecção e a dificuldade em despertar nos reeducandos o conhecimento acerca da necessidade das medidas de prevenção, já esperados para uma realidade de produção diferente da habitual. ${ }^{7,9,15}$

Nesse sentido, uma das importantes demandas para implementação do projeto foi adaptar a sala de confecção disponibilizada às normas do protocolo de boas práticas para a produção de EPI. Considerando a necessidade de manter o processo limpo, a adoção de práticas padronizadas é fundamental para que o produto possua as especificações técnicas adequadas e atue como barreira de proteção. 5,14

Ainda, dada a promulgação da RDC no 356 de 2020, a fabricação de EPI em ambientes extraordinários implicou na necessidade de se manterem os padrões de adequação dos produtos, 
Produção de aventais descartáveis em complexo prisional: ação de enfermagem no enfrent... $\mid 8$ fato que fomentou a reestruturação do ambiente de produção no local fornecido. Apesar de desafiadora, essa ação garantiu a manutenção da produção, bem como forneceu segurança à equipe operacional em termos de distanciamento social., ${ }^{2,14}$

Dentro da estrutura industrial de confecção do complexo prisional foi fornecida para produção dos aventais uma sala de confecção com aproximadamente 20 máquinas de costura industriais. A sala foi remodelada para promover o distanciamento físico entre os reeducandos; assim, a produção funcionou com pouco mais da metade da capacidade máxima, tendo em vista a redução do risco de infecção. Além disso, a reestruturação permitiu a criação dos ambientes de preparo, dobradura, embalagem e armazenamento, além da instalação de uma barreira física na entrada da sala de produção e disposição de banners com orientações sobre a técnica de higienização das mãos e limpeza do ambiente (Figura 1).

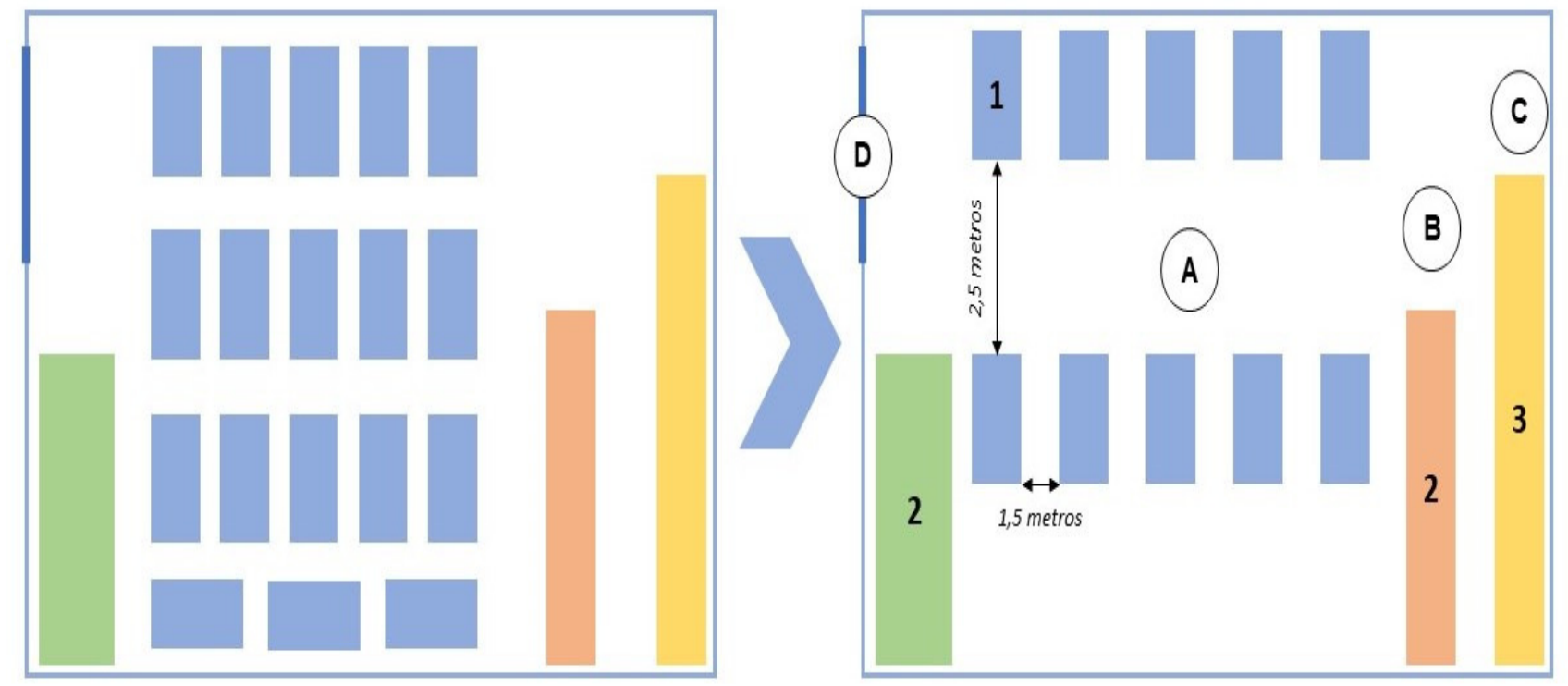

Figura 1 - Estrutura física do polo de produção de aventais do Complexo Prisional antes e após o remodelamento. A: Área de confecção; B: Área de preparo, dobradura e selagem; C: Área de armazenamento e distribuição; D: Entrada/Barreira física; 1: Máquinas de costura; 2: Bancadas para separação dos cortes (verde), preparo, dobradura e selagem (rosa); 3: Armário para armazenamento.

O processamento de produtos para a saúde implica na adoção de medidas para a manutenção do processo limpo, garantindo, por meio de infraestrutura adequada e etapas bem 
definidas, a redução do risco de infecção ao paciente. Ainda, falhas nesse processo resultam em comprometimento do material final..$^{9,11,15}$

Considerando esses pressupostos, a impossibilidade de ter uma barreira física entre a área de preparo, dobradura e selagem e a área de armazenamento e distribuição implicou na necessidade de reduzir a circulação na sala e implementar um fluxo unidirecional de produção. Dessa forma, foi possível adequar a produção às limitações de infraestrutura, mas respeitando as normativas vigentes, dada a necessidade desses insumos para o combate à pandemia., , $11,13^{13}$

Para além dos desafios impostos pelos recursos estruturais, a necessidade de trabalhar com paramentação adequada e adoção de medidas de higienização das mãos constantemente resultou em uma mudança na rotina de trabalho dos reeducandos. A partir da capacitação e do acompanhamento diário pelos pós-graduandos, houve adesão às medidas por parte da equipe a essas na rotina de trabalho.

Naturalmente, a medida de higienização das mãos era mais utilizada por profissionais e estudantes da área da saúde anteriormente à pandemia $;{ }^{19}$ entretanto, a alta transmissibilidade do SARSCoV-2 suscitou a adoção dessa medida pela população, caracterizando uma das principais medidas de prevenção a fim de quebrar a cadeia de transmissão. ${ }^{20}$ Assim, tendo em vista a finalidade da produção, a adoção de boas práticas foi fundamental para a garantia do processo limpo e da segurança da equipe.

Com foco em capacitar os reeducandos para a execução da produção, respeitando as recomendações de condições adequadas de higiene e segurança no ambiente de trabalho, ${ }^{6}$ foi realizada atividade de educação em saúde. Esta envolveu o ensino da técnica correta de higienização das mãos, em que momentos elas deveriam ser realizadas durante as etapas de produção, distanciamento físico, a etiqueta respiratória a ser seguida durante a produção, paramentação e desparamentação adequadas, limpeza e desinfecção de superfícies na área de produção, manuseio correto do TNT e demais condutas a serem realizadas no preparo, dobradura, embalagem e armazenamento da produção. Tais ações foram desenvolvidas com foco em manter o processo limpo e evitar contaminação do material. ${ }^{21}$ 
Produção de aventais descartáveis em complexo prisional: ação de enfermagem no enfrent... | 10

Destaca-se nesse processo o papel formativo e social da ação em oportunizar aos reeducandos a possibilidade de conhecer as medidas de controle de infecção necessárias para a quebra da cadeia de transmissão do vírus, considerando a vulnerabilidade já imposta a essa população no ambiente prisional.,22 Assim, com a finalidade de evitar a contaminação da matéria-prima e dos aventais durante a produção, foram adotadas medidas como limpeza diária da sala e forragem do piso com plástico antes do início da produção. Para evitar contato dos aventais com o chão durante o processo de costura, eles foram acondicionados em caixas de plástico rígido dispostas ao lado de cada máquina de costura; diariamente as caixas eram submetidas à limpeza e desinfecção com álcool 70\%.

A rotina de trabalho dos reeducandos ocorreu de forma sistematizada de acordo com o apresentado na Figura 2.

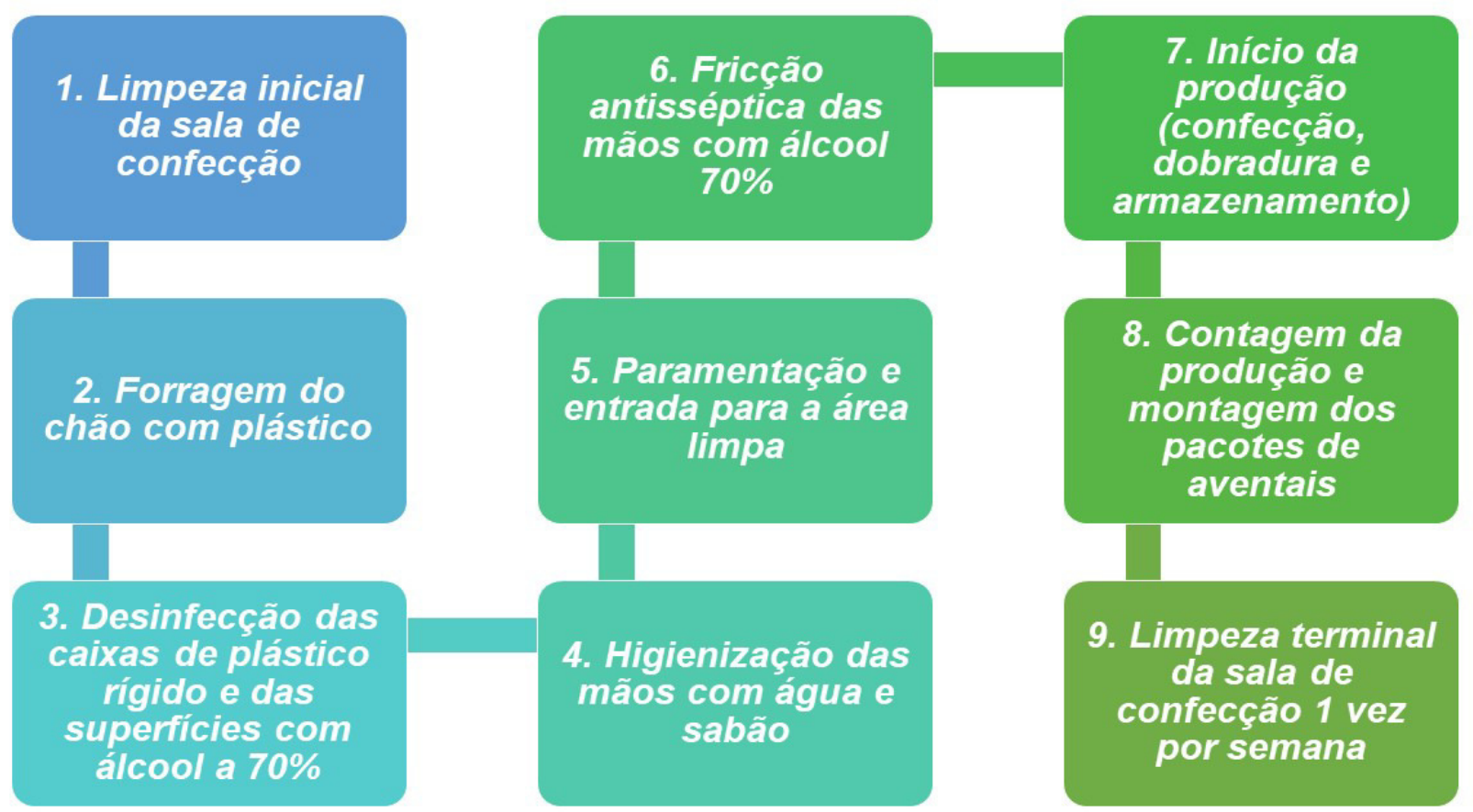

Figura 2 - Sistematização da rotina de trabalho dos reeducandos na produção de EPI. Goiânia, GO, Brasil, 2020.

Ao final do processo o avental era dobrado, acondicionado em embalagens plásticas resistentes e transportado de forma segura em containers para uma área exclusiva de guarda e distribuição na Faculdade de Enfermagem. Devido ao pequeno espaço para armazenamento da 
produção, o transporte para o centro de distribuição era realizado a cada dois dias. Seguindo essa rotina de trabalho a produção média foi de 200 aventais/dia. No centro de distribuição eram realizadas as etapas de conferência do produto, empacotamento, rotulagem, armazenamento e distribuição para os hospitais públicos do Estado de Goiás, de acordo com as necessidades de cada instituição, além de fornecimento às populações vulneráveis.

O sentimento de pertencimento dos envolvidos associado à possibilidade de contribuir para salvar vidas no momento da pandemia da COVID-19 evidenciam o papel social do projeto, da universidade e da enfermagem ao promover a interlocução entre diferentes setores da sociedade. ${ }^{12,22}$

\section{Papel da enfermagem no desenvolvimento das ações de enfrentamento à COVID-19 no complexo prisional}

Considerando os objetivos almejados pela campanha Nursing Now para a enfermagem mundial e o marco histórico do bicentenário de Florence Nightingale, marcando 2020 como o ano da profissão, ${ }^{23}$ a inserção de enfermeiros em ações de combate à pandemia tem refletido o protagonismo da categoria nas diferentes esferas da saúde e, para além disso, tem evidenciado os anseios de valorização profissional apontados em sua importância para o enfrentamento da pandemia. ${ }^{22,24}$

Assim, corroborando com outras iniciativas, ${ }^{12,18}$ a participação de docentes, discentes e voluntários foi fundamental para a execução do projeto. A experiência relatada contou com a participação de enfermeiros, estudantes da pós-graduação stricto sensu que colaboraram na supervisão da produção, desde a capacitação dos reeducandos até a entrega do material produzido.

O impacto social das ações realizadas de forma integrada entre os diversos atores do projeto refletiu no fortalecimento da enfermagem como profissão dada a visibilidade, o alcance e a integração da ação de extensão para com as esferas da sociedade. Considerando a meta global de ofertar saúde a todos até 2030 e o importante déficit de profissionais de enfermagem nas Américas, ${ }^{25}$ ações como esta promovem o reconhecimento e fortalecimento da enfermagem, contribuindo para o alcance das metas da campanha Nursing Now de desenvolvimento 
Produção de aventais descartáveis em complexo prisional: ação de enfermagem no enfrent... | 12

profissional e em todos os níveis de liderança, atuação integral e disseminação das práticas de enfermagem efetivas e inovadoras. ${ }^{23}$

Além disso, a adoção de medidas de higiene e a implementação de protocolos para a produção de EPI, bem como a adoção das medidas de biossegurança quanto à COVID-19 corroboraram com a discussão levantada pelo bicentenário de Florence Nightingale acerca de seu papel na implementação de protocolos para a redução do risco de infecção, tão atuais ainda no século XXI. ${ }^{23}$

\section{Desafios no desenvolvimento da experiência}

$\mathrm{Na}$ perspectiva da experiência, os desafios enfrentados na implementação das medidas de biossegurança foram limitadores importantes. Devido ao espaço utilizado para a produção ter como finalidade primária a confecção de roupas, sua adaptação para atender às medidas de biossegurança implicou em dificuldades na delimitação de espaços individuais para cada etapa do processo.

Assim, a equipe ficou limitada ao mínimo necessário, tendo em vista o processo de produção e a implementação das medidas de prevenção e controle da COVID-19. Cabe ressaltar que não havia pia para higiene de mãos no espaço de produção nem espaço adequado para armazenamento de matéria-prima e produtos finalizados, o que demandou uma logística para entrega de matéria-prima e dispensação no centro de distribuição.

Já na perspectiva do desenho do estudo, o caráter de relato de experiência limitou a apresentação da percepção dos reeducandos envolvidos no projeto, que pode ser fonte de investigações futuras. Entretanto, é reconhecido pelos autores o papel social das atividades desempenhadas, com destaque para os atores envolvidos, e a importância da enfermagem na condução da ação de extensão.

\section{Conclusão}

A experiência de produção de aventais descartáveis em complexo prisional para combate à COVID-19 destacou a contribuição da enfermagem na liderança de ações de saúde com 
importante impacto para a sociedade. Diante da necessidade da comunidade de produção de EPI para profissionais de saúde, a ação foi de grande relevância para os docentes, discentes e profissionais voluntários envolvidos no projeto. Além de ser uma oportunidade ímpar no contexto da pós-graduação, no desenvolvimento de conhecimentos, habilidades e atitudes, quanto a gestão, liderança, comunicação, educação em saúde e trabalho em equipe.

Apesar dos desafios, foi possível compreender o papel de ressocialização do trabalho dentro do contexto prisional, reforçando essa atividade como essencial para a manutenção do sistema. O trabalho dos reeducandos aponta para uma janela de oportunidades no que diz respeito à articulação entre a universidade e esferas do Governo, bem como à possibilidade do comprometimento social daqueles que estão sendo reinseridos na sociedade. Vislumbra-se nessa alternativa a possibilidade da melhora da empregabilidade e retomada dos papéis de cidadão e atores ativos na sociedade.

É desafiador enfrentar um cenário de pandemia que interfira direta e indiretamente nos aspectos sociais, econômicos, políticos, pessoais e de saúde de toda a população. Contudo, é papel da universidade pública devolver à sociedade a confiança que lhe é investida a partir da resolução de questões cotidianas de forma a promover a beneficência, evitando a maleficência, reforçando o papel das relações comunidade-instituição.

Contribui para o trabalho da enfermagem no âmbito social ao promover a saúde e oportunidade de profissionalização, assim como crescimento da população privada de liberdade, e também ao reconhecer a habilidade da gestão da pandemia, diante da escassez de EPI para promover segurança e qualidade de vida aos profissionais de saúde que estavam na linha de frente, além de contribuir para a redução dos gargalos produzidos pela escassez de tais insumos no contexto pandêmico, fortalecendo a visão da profissão como liderança no combate aos agravos de saúde pública.

\section{Referências}

1. Spinelli A, Pellino G. COVID-19 pandemic: perspectives on an unfolding crisis. Br J Surg. 2020 Jun;107(7):7857. doi: 10.1002/bjs.11627 
Produção de aventais descartáveis em complexo prisional: ação de enfermagem no enfrent... | 14

2. World Health Organization (WHO). Transmission of SARS-CoV-2: implications for infection prevention precautions: scientific brief, 09 July 2020 [Internet]. Geneva: World Health Organization; 2020 [cited 2020 Aug 15]. Available from: https://apps.who.int/iris/handle/10665/333114

3. Bong C-L, Brasher C, Chikumba E, McDougall R, Mellin-Olsen J, Enright A. The COVID-19 Pandemic: effects on low- and middle-income countries. Anesth Analg. 2020;131(1):86-92. doi: 10.1213/ANE.0000000000004846

4. Noronha KVMS, Guedes GR, Turra CM, Andrade MV, Botega L, Nogueira D, et al. Pandemia por COVID-19 no Brasil: análise da demanda e da oferta de leitos hospitalares e equipamentos de ventilação assistida segundo diferentes cenários. Cad Saúde Pública. 2020;36(6):e00115320. doi: 10.1590/0102-311x00115320

5. World Health Organization (WHO). Rational use of personal protective equipment for coronavirus disease (COVID-19) and considerations during severe shortages: interim guidance, 6 April 2020 [Internet]. Geneva: World Health Organization; 2020 [cited 2020 Aug 19]. Available from: https://apps.who.int/iris/handle/10665/331695

6. World Health Organization (WHO). Considerations for public health and social measures in the workplace in the context of COVID-19: annex to considerations in adjusting public health and social measures in the context of COVID-19, 10 May 2020 [Internet]. Geneva: World Health Organization; 2020 [cited 2020 Aug 19]. Available from: https://apps.who.int/iris/handle/10665/332050

7. Ranney ML, Griffeth V, Jha AK. Critical supply shortages - the need for ventilators and personal protective equipment during the Covid-19 pandemic. N Engl J Med. 2020;382(18):e41. doi: 10.1056/NEJMp2006141

8. Almeida IM. Proteção da saúde dos trabalhadores da saúde em tempos de COVID-19 e respostas à pandemia. Rev Bras Saúde Ocup. 2020;45:e17. doi: 10.1590/scielopreprints.140

9. Park C-Y, Kim K, Roth S, Beck S, Kang JW, Tayag MC, et al. Global shortage of personal protective equipment amid COVID-19: supply chains, bottlenecks, and policy implications. 2020;130:1-10. doi: 10.22617/BRF200128-2

10. Sommerstein R, Fux CA, Vuichard-Gysin D, Abbas M, Marschall J, Balmelli C, et al. Risk of SARS-CoV-2 transmission by aerosols, the rational use of masks, and protection of healthcare workers from COVID-19. Antimicrob Resist Infect Control. 2020;9(1):100. doi: 10.1186/s13756-020-00763-0

11. BRASIL. Agência Nacional de Vigilância Sanitária. Resolução de Diretoria Colegiada (RDC) no 356, de 23 de março de 2020. Dispõe, de forma extraordinária e temporária, sobre os requisitos para a fabricação, importação e aquisição de dispositivos médicos identificados como prioritários para uso em serviços de saúde, em virtude da emergência de saúde pública internacional relacionada ao SARS-CoV-2. Diário Oficial da União: Edição 56C, seção 1-Extra, Brasília, DF, p. 5, 23 mar. 2020. Disponível em: https://www.in.gov.br/en/web/dou/-/resolucaordc-n-356-de-23-de-marco-de-2020-249317437. Acesso em: 16 ago. 2020.

12. Silva MRF, Mascarenhas ALLD, Dutra MCFSG, Silva CAF, Dias NS. Reflections on extensionist and research actions in the fight against COVID-19 at the university of the state of Rio Grande do Norte. Braz J Health Rev. 2020;3(2):3622-46. doi: 10.34119/bjhrv3n2-191

13. Universidade Federal de Goiás (UFG). Projeto EPI-UFG: manual [Internet]. Goiânia: Cegraf UFG; 2020 
15 | Silva GO, Ues LV, Hoelzel CGM, Neves HCC, Mendonça KM, Ribeiro LCM

[acesso em 2020 ago 15]. $74 \quad$ p. Disponível em: https://files.cercomp.ufg.br/weby/up/1/o/Manual_ficha_catalografica_final.pdf

14. Associação Brasileira de Normas Técnicas (ABNT). NBR ISO 16693:2018 - Produtos têxteis para saúde Aventais e roupas privativas para procedimento não cirúrgico utilizados por profissionais de saúde e pacientes Requisitos e métodos de ensaio. Rio de Janeiro: ABNT; 2018. 9 p.

15. Sinha MS, Bourgeois FT, Sorger PK. Personal Protective Equipment for COVID-19: distributed fabrication and additive manufacturing. Am J Public Health. 2020 Aug;110(8):1162-4. doi: 10.2105/AJPH.2020.305753

16. Khan MM, Parab SR. Simple economical solution for Personal Protection Equipment (face mask/shield) for health care staff during COVID 19. Indian J Otolaryngol Head Neck Surg. 2020 Apr 27:1-5. doi: 10.1007/s12070020-01863-4

17. Shokrani A, Loukaides EG, Elias E, Lunt AJG. Exploration of alternative supply chains and distributed manufacturing in response to COVID-19; a case study of medical face shields. Mater Des. 2020;192:108749. doi: 10.1016/j.matdes.2020.108749

18. Panizzon M, Costa CF, Medeiros IBO. Práticas das universidades federais no combate à COVID-19: a relação entre investimento público e capacidade de implementação. Rev Adm Pública. 2020;54(4):635-49. doi: 10.1590/0034-761220200378

19. Zottele C, Magnago TSBS, Dullius AIS, Kolankiewicz ACB, Ongaro JD. Hand hygiene compliance of healthcare professionals in an emergency department. Rev Esc Enferm USP. 2017;51:e03242. doi: 10.1590/s1980220x2016027303242

20. Centers for Disease Control and Prevention (CDC). Interim infection prevention and control recommendations for healthcare personnel during the coronavirus disease 2019 (COVID-19) pandemic [Internet]. Atlanta (GA): CDC; 2020 [cited 2020 Aug 30]. Available from: https:/www.cdc.gov/coronavirus/2019ncov/hcp/infection-control-recommendations.html

21. Tipple AFV, Santos JEM, Costa DM, Bouwman BE, Goulart DMM. Reusable medical devices storage area: rethinking the shelves decontamination frequency. Ciênc Cuid Saúde [Internet]. 2020 May 4 [cited 2020 Sept 30];19:e48223. Available from: https://periodicos.uem.br/ojs/index.php/CiencCuidSaude/article/view/48223/751375150010

22. Choi KR, Jeffers KS, Logsdon MC. Nursing and the novel coronavirus: risks and responsibilities in a global outbreak. J Adv Nurs. 2020 Jul;76(7):1486-7. doi: 10.1111/jan.14369

23. Crisp N, Iro E. Nursing Now Campaign: raising the status of nurses. Lancet. 2018 Mar 10;391(10124):920-1. doi: 10.1016/S0140-6736(18)30494-X

24. Oliveira KKD, Freitas RJM, Araújo JL, Gomes JGN. Nursing Now e o papel da enfermagem no contexto da pandemia e do trabalho atual. Rev Gaúcha Enferm. 2020;42(Spec No). doi: 10.1590/1983-1447.2021.20200120

25. Pan American Health Organization (PAHO). Strategic directions for nursing in the Region of the Americas [Internet]. Washington (DC): PAHO; 2019 [cited 2020 Sept 30]. Available from: http://iris.paho.org/xmlui/bitstream/handle/123456789/50956/9789275120729_eng.pdf?sequence=1\&amp 
Produção de aventais descartáveis em complexo prisional: ação de enfermagem no enfrent... | 16

Editora Científica: Tânia Solange Bosi de Souza Magnago

Editora associada: Alexa Pupiara Flores Coelho

Fomento / Agradecimento: Não possui.

\author{
Autor correspondente \\ Luana Cássia Miranda Ribeiro \\ E-mail: luaufg@yahoo.com.br \\ Endereço: FEN - Faculdade de Enfermagem/UFG Rua 227 Qd. 68 s/n - Setor Universitário. Goiânia - Goiás - Brasil. \\ CEP: 74605-080
}

\title{
Contribuições de Autoria
}

1 - George Oliveira Silva

Concepção ou desenho do estudo/pesquisa, análise e/ou interpretação dos dados, revisão final com participação crítica e intelectual no manuscrito.

\section{2 - Larissa Viana Ues}

Concepção ou desenho do estudo/pesquisa; (2) análise e/ou interpretação dos dados (3) revisão final com participação crítica e intelectual no manuscrito).

\section{3 - Carlos Gustavo Martins Hoelzel}

Concepção ou desenho do estudo/pesquisa, análise e/ou interpretação dos dados, revisão final com participação crítica e intelectual no manuscrito.

\section{4 - Heliny Carneiro Cunha Neves}

Concepção ou desenho do estudo/pesquisa, análise e/ou interpretação dos dados, revisão final com participação crítica e intelectual no manuscrito.

\section{5 - Katiane Martins Mendonça}

Concepção ou desenho do estudo/pesquisa, análise e/ou interpretação dos dados, revisão final com participação crítica e intelectual no manuscrito.

\section{6 - Luana Cássia Miranda Ribeiro}

Concepção ou desenho do estudo/pesquisa, análise e/ou interpretação dos dados, revisão final com participação crítica e intelectual no manuscrito.

\section{Como citar este artigo}

Silva GO, Ues LV, Hoelzel CGM, Neves HCC, Mendonça KM, Ribeiro LCM. Production of disposable gowns in a prison complex: nursing action in confronting COVID-19. Rev. Enferm. UFSM. 2021 [Access: Year Month Day]; vol.11 e65: 1-16. DOI: https://doi.org/10.5902/2179769264899 